\title{
Pseudo-arthrosis repair of a posterior cruciate ligament avulsion fracture
}

\author{
Paul Hoogervorst · J. W. M. Gardeniers • \\ S. Moret-Wever · A. van Kampen
}

Received: 16 December 2009/Accepted: 4 March 2010/Published online: 20 April 2010

(C) The Author(s) 2010. This article is published with open access at Springerlink.com

\begin{abstract}
A pseudo-arthrosis repair of a 4-year-old bony avulsion fracture of the PCL using a minimally invasive technique, screw fixation, and bone grafting is reported. The case presented seems to be rather unique due to the fragment size and the approach for pseudoarthrosis repair. There was a good functional result following minimally invasive pseudo-arthrosis repair of a posterior cruciate ligament avulsion fracture. There are no previous reports of similar pseudo-arthrosis repairs, and other authors report good results of delayed refixation of PCL avulsion fractures. Therefore, refixation and pseudo-arthrosis repair should be considered as a viable treatment.
\end{abstract}

Keywords Posterior cruciate ligament · Avulsion ·

Pseudo-arthrosis $\cdot$ Repair $\cdot$ Screw fixation

\section{Introduction}

The posterior cruciate ligament (PCL) plays a major role in knee stabilization. It acts as a primary restraint against posterior displacement and, in concert with the anterior cruciate ligament (ACL), to regulate external rotation of the knee during extension [3].

The most commonly reported mechanism of injury to the PCL is a posteriorly directed blow to the anterior aspect

P. Hoogervorst $(\bowtie) \cdot$ J. W. M. Gardeniers · S. Moret-Wever ·

A. van Kampen

Department of Orthopaedic Surgery,

St Radboud University Nijmegen Medical Centre,

Theodoor Craanenlaan 7, 6525 GH Nijmegen, The Netherlands

e-mail: p.hoogervorst@orthop.umcn.nl of the proximal tibia with the knee flexed at $90^{\circ}$ [14]. Another mechanism is sudden hyperextension in conjunction with associated varus or valgus force or hyperflexion of the knee [5].

Sports injury and motor vehicle accidents account for the majority of PCL injuries [10]. Clinical examination shows a posterior sag and a positive posterior drawer test if the PCL is ruptured or avulsed. In case of avulsion, radiological examination will reveal a bony fragment at the tibial attachment of the PCL.

Avulsion of the tibial insertion of the PCL is believed to be an uncommon injury. A non-displaced bony avulsion can be treated conservatively with a plaster cast, holding the knee flexed and the tibia pulled forward to diminish tension on the PCL. There is general consensus that a displaced bony PCL avulsion should be surgically reduced and fixed immediately to stabilize the knee joint and prevent non-union [10].

All publications on the repair of acute bony avulsion of the tibial attachment of the PCL report excellent clinical results $[1,2,4,6,9-12,14]$.

Information on the treatment of bony PCL avulsions past the acute phase is rare. Trickey states that surgical repair of an avulsion the tibial attachment of the PCL after 3 weeks is not successful [13]. However, Meyers and Torisu dispute this statement $[10,12]$. There is one report in the literature of a reduction and screw fixation combined with posterolateral stabilization of a 4.5-yearold bony avulsion of the PCL with good clinical result [7].

To the authors' knowledge, there is no report of pseudoarthrosis repair of a PCL avulsion fracture. We report the case of a pseudo-arthrosis repair of a 4-year-old bony avulsion fracture of the PCL using a minimally invasive technique, screw fixation, and bone grafting. 


\section{Case report}

A 57-year-old woman presented to the orthopaedic outpatient clinic. Four years prior, she suffered from distorsion trauma of her left knee during a skiing accident. At the time, the knee was examined both physically and radiologically but the diagnosis of an avulsion fracture of the tibial eminence was not made. In the years following the initial trauma, the patient reported continued pain and instability. The patient described a "clicking" sensation while walking and posterior pain on weight bearing. She also reported a tired feeling of the entire lower left extremity. She wore a brace, which reduced but failed to entirely relieve her symptoms. Physical examination showed a healthy appearing woman with slight varus alignment. The left knee had a full range of motion without any apparent instability but did show a slight posterior sag. Meniscal testing showed no abnormalities.

Standard lateral and posterior-anterior radiographs revealed an evident pseudo-arthrosis with a radiolucent and sclerotic line under the posterior tibial eminence. The avulsed fragment measured $32 \mathrm{~mm} \times 24 \mathrm{~mm} \times 12 \mathrm{~mm}$ (Fig. 1).

Magnetic resonance imaging and computed tomography showed pseudo-arthrosis of a large bony avulsion of the tibial attachment of the PCL extending to both the medial and lateral tibial plateau The magnetic resonance imaging also showed an intact PCL and did not reveal any signs of concomitant intra-articular injuries of the knee (Fig. 2). Bone scintigraphy demonstrated increased activity at the proximal left tibia.

It was concluded that the patient's complaints could be caused by the pseudo-arthrosis of a bony avulsion of the tibial attachment of the PCL; this was deemed an appropriate indication for pseudo-arthrosis repair.

\section{Surgical technique}

The patient was positioned supine and the lower extremity held in $30^{\circ}$ flexion. A single $2-\mathrm{g}$ dose of intravenous ceftazidime was administered. Under fluoroscopic control, an entrance point for a $10-\mathrm{mm}$ cannulated drill along the medial aspect of the proximal tibia was located. A stabincision was made, and the cannulated drill was inserted and directed cranially and posteriorly towards the centre of the pseudo-arthrosis until the tip of the drill was located just subchondral at the lateral tibial plateau. Position of the drill was verified by fluoroscopy (Fig. 3).

The drill and the collected tissue, consisting of normal cancellous bone and fibrous tissue, were removed. A similar manoeuvre was performed, only now the $10 \mathrm{~mm}$ cannulated drill was directed more posteriorly in order to access more of the pseudo-arthrosis. The fibrous tissue was separated from the healthy cancellous bone for microscopic evaluation (Fig. 3).
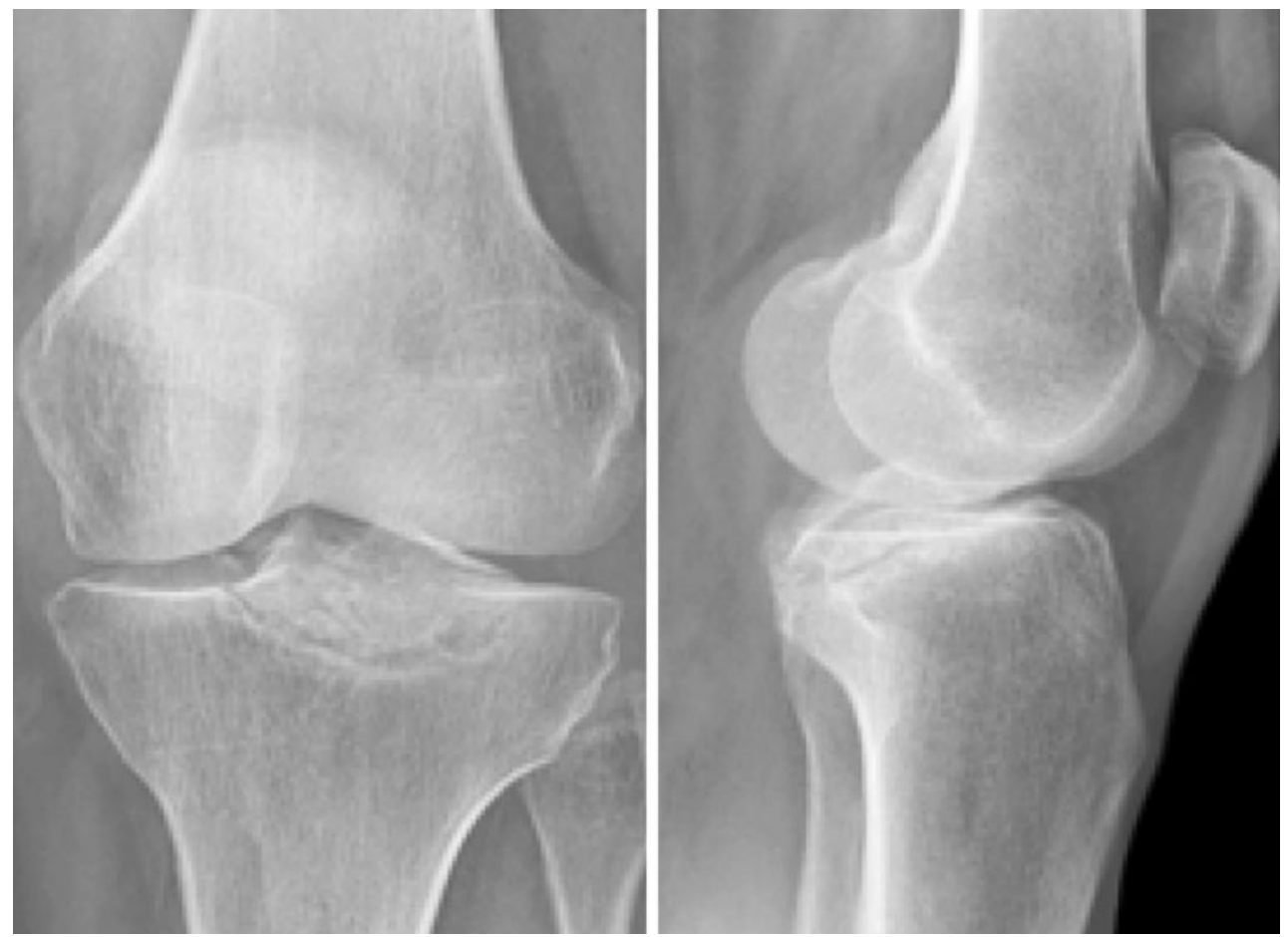

Fig. 1 Standard radiographs showing an evident pseudo-arthrosis of the posterior tibial eminence 

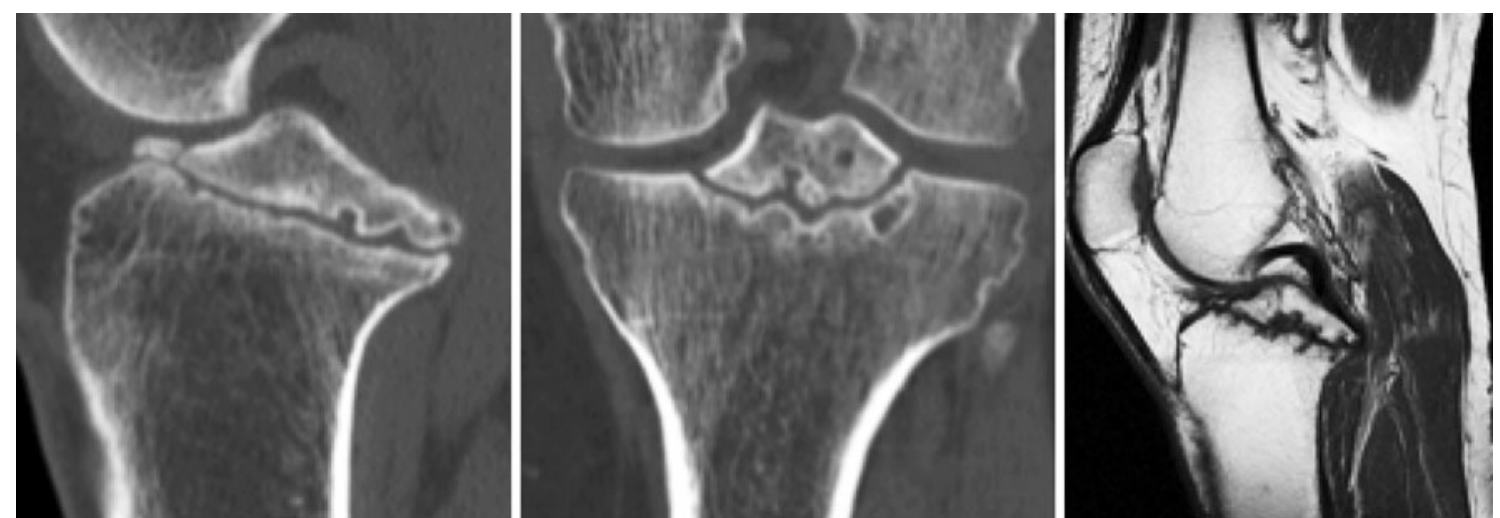

Fig. 2 Computed tomography revealing a large bony avulsion of the tibial attachment of the PCL. Magnetic resonance imaging shows an intact PCL
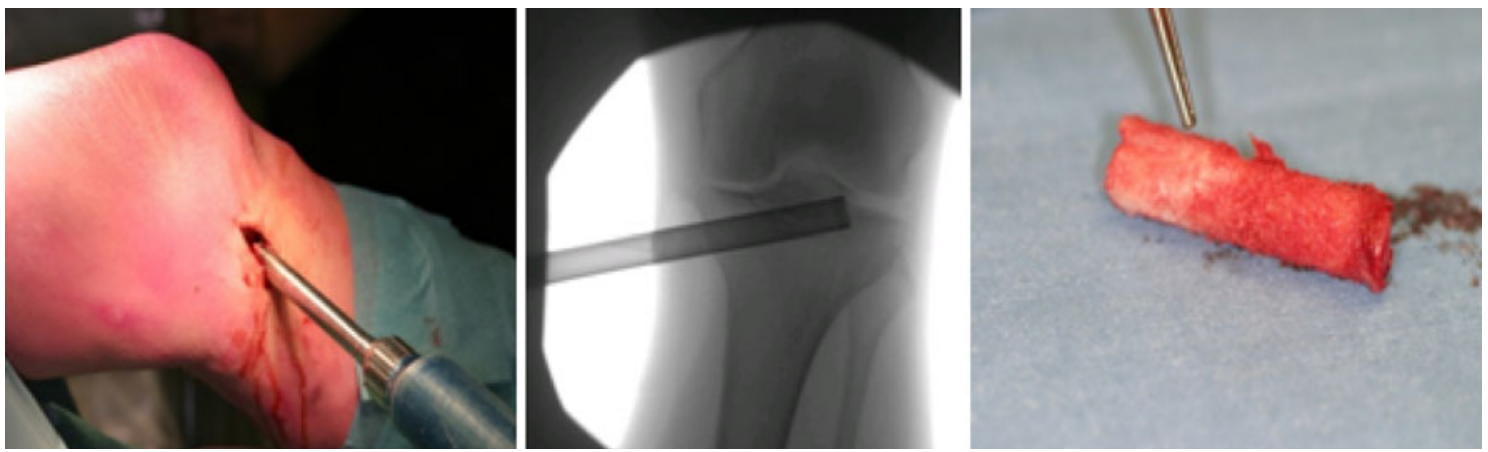

Fig. 3 Positioning of the cannulated drill and its fluoroscopic verification. Removed pseudo-arthrosis shows the fibrous tissue and healthy cancellous bone

Using a curette through the drill-hole, the remaining pseudo-arthrosis was removed under fluoroscopic control (Fig. 4). A combination of the removed cancellous bone mixed with homologous bone was used to fill the defect created by the cannulated drill. Fluoroscopic imaging showed good positioning of the bone grafting. A tibial ACL guide was used to guide Kirschner wires for two 4.5$\mathrm{mm}$ cannulated partially threaded screws. These screws were used to lag and stabilize the bony fragment.
There was a good fixation and compression of the avulsed fragment. Radiographic evaluation demonstrated good positioning of the screws with all screw threads in the avulsed bony fragment (Fig. 5).

Postoperatively, the patient was mobilized immediately with a full leg cylinder plaster cast. There was no weight bearing for the first 6 weeks followed by full weight bearing without the plaster cast. Mobilization was guided by a physical therapist. Three months after surgery, the
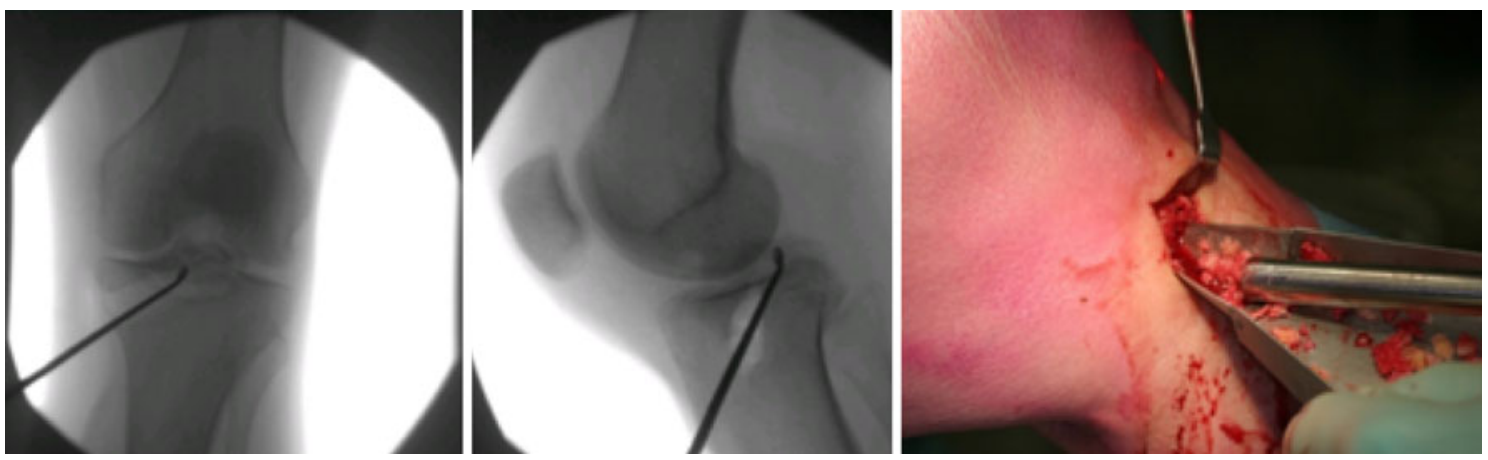

Fig. 4 Removal of the remaining pseudo-arthrosis using a curette and bone grafting of the defect created by the cannulated drill 

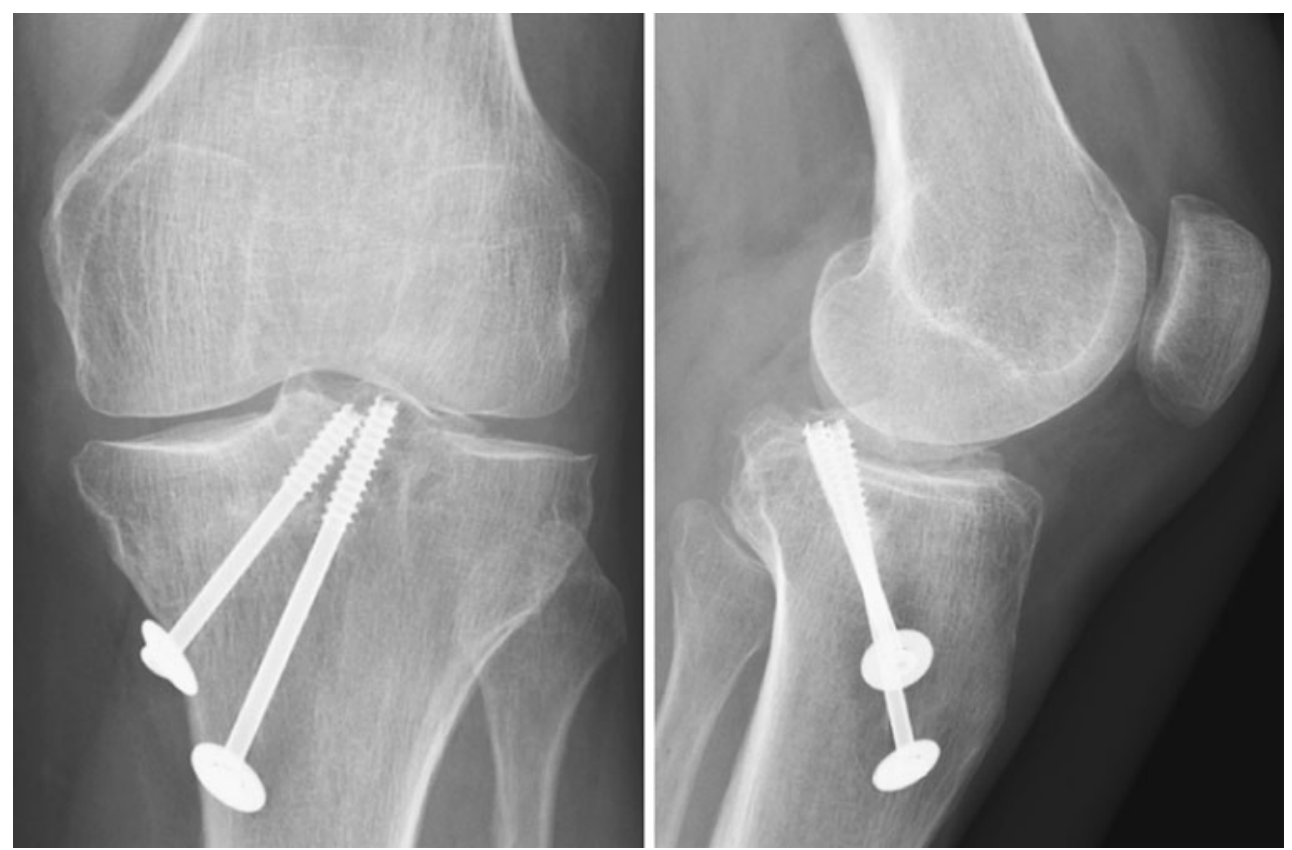

Fig. 5 Standard radiographs showing positioning of the lag screws and complete disappearance of the pseudo-arthrosis of the bony avulsion of the tibial insertion of the PCL

patient was satisfied with the result of the surgical intervention. The complaints of a "clicking" sensation and posterior pain on weight bearing were completely resolved. Physical examination showed no posterior sag or anteriorposterior instability. Radiographs showed a complete disappearance of the pseudo-arthrosis of the bony avulsion of the tibial insertion of the PCL and good incorporation of the bone graft (Fig. 5).

\section{Discussion}

In this case, there was a good functional result following minimally invasive pseudo-arthrosis repair of a posterior cruciate ligament avulsion fracture.

In displaced bony PCL avulsion injuries, there is general consensus that surgical reduction and fixation in the acute phase is indicated to stabilize the joint and prevent nonunion [10]. However, proper treatment of bony PCL avulsions past the acute phase is uncertain. Trickey states that surgical repair of an avulsion the tibial attachment of the PCL after 3 weeks is not successful [13]. Kim et al. reported two patients with non-union tibial avulsion fractures who underwent surgical reduction and fixation 19 and 20 months after the causal trauma. Both were described to have grade $\mathrm{C}$ (abnormal) function postoperatively according to the assessment with the IKDC form [8].

In contrast, Torisu reported good results in three patients with delayed repair of bony PCL avulsion fractures; he stated that late repair is recommended [12]. Meyers [10] reported two patients with delayed treatment of displaced PCL avulsion fractures operated three and 6 months after the initial trauma with good functional results. Jung et al. reported reduction and screw fixation of a 4.5-year-old displaced PCL avulsion fracture. Using a posterior approach and single screw fixation, good stability and function of the knee was obtained [7].

The case presented here seems to be rather unique due to the fragment size and the approach for pseudo-arthrosis repair. In this case of a 4-year-old pseudo-arthrosis, it was hypothesized that it would be necessary to remove the pseudo-arthrosis tissue to achieve a solid bone union. The removal of the pseudo-arthrosis tissue and the filling with homologous bone was deemed easier and safer using a medio-anterior rather than a posterior approach. A minimally invasive technique was used to reduce soft tissue damage and reduce the risk of injury to the popliteal neurovascular branch. Furthermore, a medio-anterior approach was preferred over a posterior one for optimal screw fixation in the centre of the avulsed bony fragment. Partially threaded screws were chosen to achieve a rigid and stable fixation with compression between the bony avulsion fragment and the proximal tibia.

There are no previous reports of similar pseudo-arthrosis repairs, but good results of delayed refixation of PCL avulsion fractures have been described. Therefore, refixation and pseudo-arthrosis repair should be considered as a viable treatment. 
Open Access This article is distributed under the terms of the Creative Commons Attribution Noncommercial License which permits any noncommercial use, distribution, and reproduction in any medium, provided the original author(s) and source are credited.

\section{References}

1. Bonin N, Jeunet L, Obert L, Dejour D (2007) Adult tibial eminence fracture fixation: arthroscopic procedure using K-wire folded fixation. Knee Surg Sports Traumatol Arthrosc 15:857862

2. Deehan DJ, Pinczewski LA (2001) Arthrosopic reattachment of an avulsion fracture of the tibial insertion of the posterior cruciate ligament. Arthroscopy 17:422-425

3. Detenbeck LC (1974) Function of the cruciate ligaments in knee stability. Am J Sports Med 2:217-221

4. Espejo-Baena A, Lopez-Arevalo R, Urbano V, Montanez E, Martın F (2000) Arthoscopic repair of the posterior cruciate ligament: Two techniques. Arthroscopy 16:656-660

5. Fowler PJ, Messieh SS (1987) Isolated posterior cruciate ligament injuries in athletes. Am J Sports Med 15:553-557

6. Horas U, MeissnerSA, Heiss C, Schnettler R (2009) Arthoscopic fixation of posterior cruciate ligament avulsion fractures: a new minimally invasive technique. Knee Surg Sports Traumatol Arthrosc. doi:10.1007/s00167-009-0937-3
7. Jung TM, Hoher J, Weiler A (2006) Screw fixation of a $4 \frac{1}{1} 2$-yearold PCL avulsion injury. Knee Surg Sports Traumatol Arthrosc $14: 469-472$

8. Kim SJ, Shin SJ, Choi NH, Cho SK (2001) Arthroscopically assisted treatment of avulsion fractures of the posterior cruciate ligament from the tibia. J Bone Joint Surg 83A:698-708

9. Littlejohn SG, Geissler WB (1995) Arthroscopic repair of a posterior cruciate ligament avulsion. Arthroscopy 11:235-238

10. Meyers MH (1975) Isolated avulsion fractures of the tibial attachment of the posterior cruciate ligament of the knee. J Bone Joint Surg 57-A:669-672

11. Shino K, Nakata K, Mae T, Yamada Y, Shiozaki Y,Toritsuka Y (2003) Artroscopic fixation of tibial bony avulsion of the posterior cruciate ligament. Arthroscopy. doi:10.1053/jars.2003. 50062

12. Torisu $T$ (1977) Isolated avulsion fractures of the tibial attachment of the posterior cruciate ligament. J Bone Joint Surg 59A:68-72

13. Trickey EL (1980) Injuries to the posterior cruciate ligament: diagnosis and treatment of early injuries and reconstruction of late instability. Clin Orthop Relat Res 147:76-81

14. Veselko M, Saciri V (2003) Posterior approach for arthroscopic reduction and antegrade fixation of avulsion fracture of the posterior cruciate ligament from the tibia with cannulated screw and washer. Arthroscopy 19:916-921 